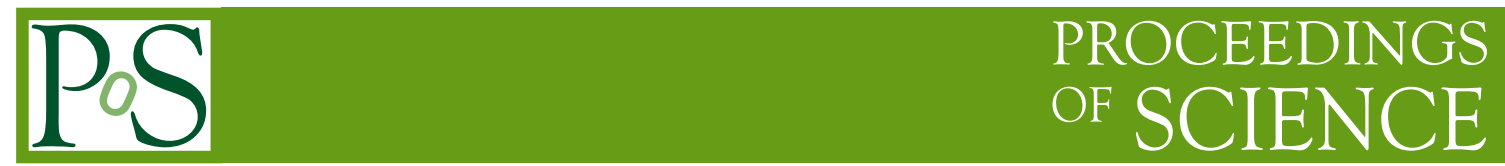

\title{
Rare B Decays Potential of SuperB
}

\author{
Alejandro Pérez \\ Laboratoire de l'Accélérateur Linéaire (LAL) \\ E-mail: perez@lal.in2p3.fr \\ On behalf of the SuperB Collaboration
}

The study of rare B-decays at SuperB provides unique opportunities to understand the Standard Model (SM) and constrains new physics (NP). It is discussed the new physics potential of the $B \rightarrow K v \bar{v}$ and $B \rightarrow K^{*} v \bar{v}$ system from the proposed SuperB experiment with $75 \mathrm{ab}^{-1}$ of data (5 nominal years of data taking).

35th International Conference of High Energy Physics - ICHEP2010,

July 22-28, 2010

Paris France 


\section{Introduction}

Rare B decays with a $v \bar{v}$ pair in the final state are interesting probes of NP, since they allow one to transparently study contributions to $Z$ and electroweak penguins. Furthermore, since the neutrinos escape the detector unmeasured, the $B \rightarrow K^{(*)}+E_{\text {miss }}$ channel can also contain contributions from other light SM-singlet particles substituting the neutrinos in the decay. The $b \rightarrow s v \bar{v}$ decays are particularly interesting as it is possible to formulate model-independent phenomenological analysis. Out of the $B \rightarrow K v \bar{v}$ and $B \rightarrow K^{*} v \bar{v}$ decays, there are three observables accessible: the corresponding branching ratios and the $K^{*}$ longitudinal polarization fraction $\left\langle F_{L}\right\rangle$ from $B \rightarrow K^{*} v \bar{v}$ decays. These three observables only depend on two combinations of the Wilson coefficients $C_{L}^{V}$ and $C_{R}^{v}[1]$ through the variables $\varepsilon=\frac{\sqrt{\left|C_{L}^{V}\right|^{2}+\left|C_{R}^{v}\right|^{2}}}{\left.\mid C_{L}^{v}\right)^{S M} \mid}$ and $\eta=\frac{-\operatorname{Re}\left(C_{L}^{v} C_{R}^{v *}\right)}{\left|C_{L}^{v}\right|^{2}+\left|C_{R}^{V}\right|^{2}}$, ( $\eta$ lies in the range $\left.\left[-\frac{1}{2}, \frac{1}{2}\right]\right)$. The discussed observables can be expressed in terms of $\varepsilon$ and $\eta$ as follows,

$$
\begin{aligned}
\operatorname{Br}\left(B \rightarrow K^{*} v \bar{v}\right) & =6.8 \times 10^{-6}(1+1.31 \eta) \varepsilon^{2}, \\
\operatorname{Br}(B \rightarrow K v \bar{v}) & =4.5 \times 10^{-6}(1-2 \eta) \varepsilon^{2}, \\
\left\langle\mathrm{~F}_{L}\left(B \rightarrow K^{*} v \bar{v}\right)\right\rangle & =0.54 \frac{(1+2 \eta)}{(1+1.31 \eta)} .
\end{aligned}
$$

Table 1 shows the SM predictions and current experimental upper bounds on branching ratios $(\mathrm{Br})$. The experimental bounds on the $\mathrm{Br}$ can then be translated in excluded areas on the $\varepsilon-\eta$ plane (green area on the rightmost plot of figure 1), where the SM corresponds to $(\varepsilon, \eta)=(1,0)$.

\begin{tabular}{lcc}
\hline Observable & SM prediction & Experiment \\
\hline $\operatorname{Br}(B \rightarrow K v \bar{v})$ & $\left(6.8_{-1.1}^{+1.0}\right) \times 10^{-6}[1]$ & $<80 \times 10^{-6}[3]$ \\
$\operatorname{Br}\left(B \rightarrow K^{*} v \bar{v}\right)$ & $(4.5 \pm 0.7) \times 10^{-6}[1]$ & $<14 \times 10^{-6}[4]$ \\
$\left\langle\mathrm{F}_{L}\left(B \rightarrow K^{*} v \bar{v}\right)\right\rangle$ & $0.54 \pm 0.01[1]$ & - \\
\hline
\end{tabular}

Table 1: SM predictions and experimental $90 \%$ C.L. upper bounds for the three $B \rightarrow K^{(*)} v \bar{v}$ observables.

\section{The experimental technique and Expectations for SuperB}

The recoil technique has been developed to search for rare B decays with undetected particles, like neutrinos, in the final states. Its consists on the reconstruction of one of the two B mesons $\left(B_{\text {tag }}\right)$, produced through the $e^{+} e^{-} \rightarrow \Upsilon(4 S) \rightarrow B \bar{B}$ resonance, in a high purity hadronic or semileptonic final states, allowing to build a pure sample of $B \bar{B}$ events. Having identified the $B_{\text {tag }}$, everything in the rest of the event (ROE) belongs by default to the signal B candidate $\left(B_{\text {sig }}\right)$, and so this technique provides a clean environment to search for rare decays. In this analysis, the $B_{\text {tag }}$ is reconstructed in the hadronic modes $B \rightarrow D^{(*)} X$, where $X=n \pi+m K+p K_{S}^{0}+q \pi^{0}(n+m+p+q<$ 6), or semi-leptonic modes $B \rightarrow D^{(*)} \ell v,(\ell=e, \mu)$. In the search for $B \rightarrow K v \bar{v}\left(B \rightarrow K^{*} v \bar{v}\right)$, the signal is given by a single track identified as a kaon (a $K^{*}$ reconstructed in the $K^{* 0} \rightarrow K^{+} \pi^{-}$, $K^{*+} \rightarrow K^{+} \pi^{0} / K_{S}^{0} \pi^{+}$modes) in the ROE.

Even though the expected SuperB [2] increase in the instantaneous luminosity of a factor 100 already promises significant improvements on the before mentionned rare decays, additional activities for detector optimization are currently ongoing. The baseline SuperB detector configuration 
is very similar to $\mathrm{BaBar}$ but the boost $(\beta \gamma)$ is reduced from 0.56 to 0.28 . This boost reduction increases the geometrical acceptance and so the reconstruction efficiency. Additionally, it is considered the inclusion of a highly performant particle identification device (Fwd-PID) based on time-of-flight measurements in the forward region.

The SuperB fast simulation has been used to produce signal samples in the before mentionned detector configurations, BaBar, SuperB base-line and SuperB+Fwd-PID. This test showed a 15\% to $20 \%$ increase in efficiency using the SuperB+Fwd-PID configuration with respect to the BaBar setup, depending on the final state, mainly due to the boost reduction. For the time being no generic $B \bar{B}$ samples has being produced. To be conservative it has been assumed that the background efficiency increases by the same factor as the signal in such a way that the signal to background ratio $(S / B)$ stays constant. This global increase in efficiency provides a gain on $S / \sqrt{(S+B)}$, which would be interpreted as the signal significance for a cut and count analysis. The $S / \sqrt{(S+B)}$ ratio, for both $B \rightarrow K v \bar{v}$ and $B \rightarrow K^{*} v \bar{v}$ modes, as a function of the integrated luminosity for the three detector configurations is shown in the left and middle plots of figure 1 (BaBar (solidblack), SuperB (dotted-black) and SuperB+Fwd-PID (solid-red)). A sensitivity of 15\% (17\%) are expected for $B \rightarrow K v \bar{v}\left(B \rightarrow K^{*} v \bar{v}\right)$ at $75 \mathrm{ab}^{-1}$. The rightmost plot of figure 1 shows the constraint at $68 \%$ (blue) and $95 \%$ (red) in the $(\varepsilon, \eta)$ plane for the expected sensitivities on $\operatorname{Br}(B \rightarrow K v \bar{v})$ and $\operatorname{Br}\left(B \rightarrow K^{*} v \bar{v}\right)$ at $75 \mathrm{ab}^{-1}$.
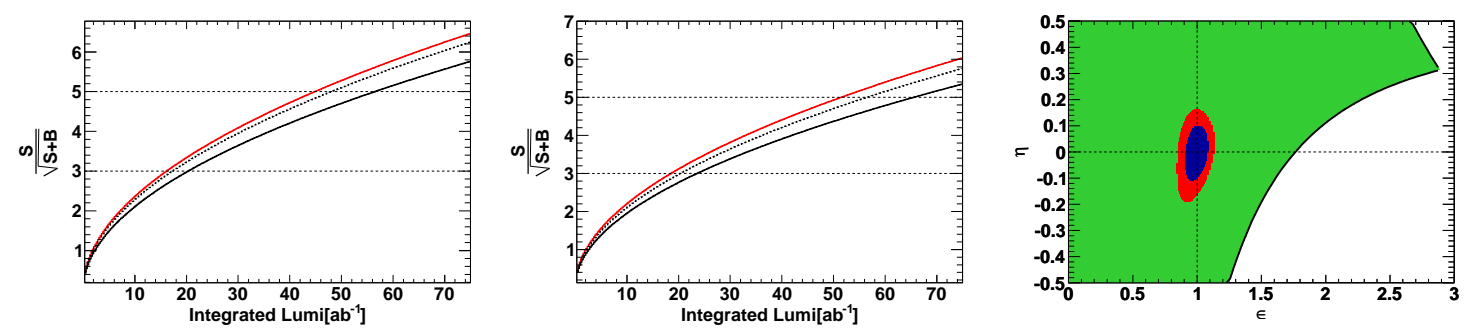

Figure 1: Expected sensitivities for the $\operatorname{Br}(B \rightarrow K v \bar{v})$ (left) and $\operatorname{Br}\left(B \rightarrow K^{*} v \bar{v}\right)$ (middle) as a function of the integrated luminosity; and expected constraint on the $(\varepsilon, \eta)$ plane for the measurement of the before mentioned branching ratios at $75 \mathrm{ab}^{-1}$ (right).

In summary, it has been investigated the reach of SuperB in the search of the $B \rightarrow K^{(*)} v \bar{v}$ decays with both the hadronic and semi-leptonic techniques. Preliminary results based on the SuperB fast simulation have shown a 15 to $25 \%$ increase in the global efficiency with respect to Babar. It has also been shown that SuperB will allow an unprecedent reduction of the NP parameter space, $(\varepsilon, \eta)$ plane, given the expected sensitivities at $75 \mathrm{ab}^{-1}$ of data.

\section{References}

[1] W. Altmannshofer, A. J. Buras, D. M. Straub, and M. Wick, JHEP 04, 022 (2009), 0902.0160.

[2] SuperB Collaboration, M. Bone et al., arXiv:0709.0451 [hep-ex].

[3] BABAR Collaboration, B. Aubert et. al., Phys. Rev. D78 (2008) 072007, 0808.1338.

[4] BELLE Collaboration, K. F. Chen et. al., Phys. Rev. Lett. 99 (2007) 221802, 0707.0138.

[5] ALEPH Collaboration, R. Barate et. al., Eur. Phys. J. C19 (2001) 213-227, 0010022. 